\title{
Pricing and hedging guaranteed returns on mix funds
}

\author{
M.H. Vellekoop ${ }^{\mathrm{a}, *}$, A.A. Vd Kamp ${ }^{\mathrm{b}}$, B.A. Post ${ }^{\mathrm{b}}$ \\ a FELab and Department of Applied Mathematics, University of Twente, P.O. Box 217, \\ 7500 AE Enschede, The Netherlands \\ ${ }^{\mathrm{b}}$ Ernst \& Young Actuaries, Euclideslaan 1, Utrecht, The Netherlands
}

Received May 2005; received in revised form December 2005; accepted 23 December 2005

\begin{abstract}
In this paper we propose a valuation and hedging strategy for a guaranteed minimal rate of return on a mix fund, which participates in both bonds and stocks. For the case where a fixed amount of money is invested, we show that a European put option on the mix fund replicates the cash flows of this guarantee at all times and using the arbitrage-free pricing methodology, the market value of the guarantee can be obtained explicitly. Using historical data, we show that modeling the correlation between equity and bond returns is of fundamental importance when the stochastic nature of the term structure of interest rates is taken into account. For this model we define a hedging strategy which shows how the dependency of the option on the changing yield of the bond fund can be hedged away using mix fund contracts. We also show how Monte Carlo methods can be used to analyze the case where the guarantee is given on periodically invested fixed amounts of money instead of one single payment.
\end{abstract}

(C) 2006 Elsevier B.V. All rights reserved.

JEL classification: G13; C15

IEM Subject Category: IE43; IE53

IEM Branch Category: IB10

Keywords: Contingent claim pricing; Mix funds; Correlation effects; Embedded options

\section{Introduction}

In many life insurance products an embedded option is an integrated part of the contract. A common life insurance product is Unit-Linked (UL) life insurance, with a minimal rate of return guarantee as the embedded option. This contract gives the policy holder the possibility to invest the insurance premium in a (predetermined) investment fund with a guaranteed minimal return at maturity. In many occasions the concerning investment fund consists of different assets such as equity, fixed-income and real estate. Such an investment fund is also known as a mix fund or mixed fund.

A UL life insurance contract where the investment portfolio is such a mix fund gives the policy holder the right to sell, at the maturity date, the net asset value of the mix fund for the guaranteed value to the insurer. All things being equal, it is obvious that for a policy holder a UL life insurance with an embedded rate of return guarantee is more valuable than a similar contract without the guarantee. Traditionally this guarantee has not been priced separately from

\footnotetext{
* Corresponding author. Tel.: +31 53489 2087; fax: +31 534893800 .

E-mail address: m.h.vellekoop@ math.utwente.nl (M.H. Vellekoop).
} 
the rest of the insurance premium and as a result the insurer runs a risk without being compensated for this. This clearly shows that in terms of expected risk and return on an investment this guarantee should have a price and may require a certain extra reservation.

In the past, 'free' embedded options such as minimal rate of return guarantees have not been a real problem for the insurance providers, since the market nearly always outperformed the guaranteed rate. But due to recent economic events such as the decreasing stock prices and declining interest rates, these guarantees are becoming more and more valuable. Already on many occasions insurance companies executives have been surprised by the financial costs of embedded options, so a better understanding of their value and associated risk seems to be needed. If the market prices of the embedded minimal rate of return guarantees were known, insurance providers would be able to charge policy holders for the risks they incur. Moreover, recent accounting standards such as IFRS increasingly require fair valuation of financial instruments, often including a clear separation between the embedded options in insurance contracts and those contracts themselves. Determining the value of embedded options in today's insurance products is therefore a major concern for insurance companies (Ernst \& Young, 2002).

If we take a closer look at the position of the policy holder of the UL contract for a single fixed invested amount of money, we see that the payoff he will receive can be realized by investing the insurance premium in the mix fund and buying (taking a long position in) a European put option on that mix fund. The insurer can then be seen as the one writing (taking a short position in) this put option. At maturity, the cash flow of a specific European put option on the mix fund replicates the minimal rate of return guarantee. For the European put option as well as for the guarantee this is the only cash flow that occurs. So preserving the arbitrage-free concept, the market value of the guarantee must be the same as that of the put option at all times. Moreover, and this is at least as important, there must exist a self-financing strategy which invests dynamically in available assets (starting with the money obtained as a price for writing the guarantee), and which replicates the payoff of the guarantee exactly, thus making it riskless to write it.

Since mix funds invest in both stocks and bonds, they are expected to be less volatile than pure stock funds due to a diversification effect, and this should make the price of a guaranteed minimal rate of return contract lower than comparable contracts which invest in stock only. However, to quantify this effect we will need to model the correlation between stock and bond funds since this will significantly influence the mentioned diversification effect. The distribution of the rate of return in a mix fund will combine features of the rates of return of both stock and fixed income funds. Using the standard Black-Scholes model to price derivative contracts which have a mix fund as the underlying asset may therefore constitute an oversimplification and a more subtle approach is therefore needed.

In this paper we propose a method to price and hedge a guaranteed return at maturity on an investment in a mix fund. We will then show how key variables, such as the correlation between stocks and bonds and the duration of the bonds in the mix fund, influence the value and hedging strategies for the guarantee. We use stochastic interest rates which are modeled by the Hull-White extension of the Vasiček short rate model, since the assumption of a constant interest rate is clearly unrealistic for long-term guarantees (Vasiček, 1977; Fabozzi, 1996). Moreover, instead of the usual technicalities associated with change of numeraire techniques to price and hedge options, we give a completely self-contained derivation, which should be of use to practitioners as well.

In many UL premium linked guarantee products, guarantees are not given for the rate of return on one single investment at one single point in time, but on the total rate of return generated by periodically invested fixed amounts of money. This makes the pricing of such a guarantee much more interesting. An extensive analysis of this case has been given in the excellent paper by Schrager and Pelsser (2004) who generalize certain lower and upper bounds on Asian option prices obtained by Nielsen and Sandmann (2002) and Thompson (1999) to approximate the value of the guarantee when the investment policy is such an annuity. Such approximations are necessary, since explicit closed-form formulas can no longer be given. The same is true for our case (a mix fund instead of the stock fund treated in their work) but we will show how our model for a mix fund can be easily used to treat this more complicated case too using Monte Carlo methods, and in particular we will speed up the calculations considerably using the explicit formulas we will derive for the easier single time investment case.

Our paper is structured in the following way. In the next section we will investigate some empirical mix fund data from the Dutch market that will help us in formulating modeling assumptions for the stochastic dynamics of the mix fund in Section 3. In Section 4 a valuation formula and the corresponding hedging strategy are derived, which is then tested in simulation studies in Section 5. In Section 6 we use Monte Carlo simulation to determine the fair value of the guarantee in case of periodic investments in the mix fund instead of a single one. We end with some conclusions and suggestions for further research. 


\section{Motivation}

The most popular model to price European options on equity is the Black-Scholes model, which assumes that the equity price process $\left\{S_{t}, t \geq 0\right\}$ follows Geometric Brownian Motion, i.e.

$$
\frac{\mathrm{d} S_{t}}{S_{t}}=\mu \mathrm{d} t+\sigma \mathrm{d} W_{t}
$$

on a filtered probability space $\left(\Omega, \mathcal{F},\left(\mathcal{F}_{t}\right)_{t \geq 0}, P\right)$ which is such that under $P$, the process $\left\{W_{t}, t \geq 0\right\}$ is an $\mathcal{F}_{t}$-adapted Brownian Motion. The constant $\sigma>0$ is assumed to be fixed and known while the fixed value $\mu \in \mathbb{R}$ does not need to be known explicitly. Under this model, future stock prices will then be lognormally distributed. Often an independent bank account is modeled as $B_{t}=B_{0} \mathrm{e}^{r t}$ with a fixed and known riskfree rate of interest $r>0$. Correlation between this fixed income instrument and stocks is thus neglected.

But this may not always be a reasonable assumption. A widely known phenomenon in the financial markets is the 'flight to quality'. Bonds are used as a safe haven by investors when the stock market becomes very volatile, implying correlation between these two investment opportunities. Prior research confirms the fact that bond and stock returns are indeed correlated, and it is very likely that correlation also exists on the level of bond and stock funds.

To investigate the characteristics of mix funds, five different mix funds in the Dutch market have been selected. All five mix funds invest in a single stock fund and a single bond fund and pursue a distribution of 50\% in each of these two funds. For each bond fund the current modified duration is approximately 5.0. We are especially interested in the correlation between the return of the bond fund and the stock fund for each of these five mix funds. In order to determine the sample correlation coefficients, we had historical monthly prices of the funds at our disposal. The results are shown in the table below. All data concern the period December 2000-December 2004 (source: Datastream).

\begin{tabular}{ll}
\hline Fund & $\hat{\rho}_{r_{s}, r_{b}}$ \\
\hline A & -0.302 \\
B & -0.244 \\
C & -0.404 \\
D & -0.366 \\
E & -0.534 \\
\hline
\end{tabular}

The correlation coefficients are negative, as we would expect due to the earlier mentioned phenomenon of 'flight to quality'. The results show remarkably high correlations between the bond and the stock fund, which are all significant at a 5\% level apart from the value for fund $B$. That value is significant for a one-sided test, where one tests $\rho=0$ against $\rho<0$, but not for a two-sided test, where one tests $\rho=0$ against $\rho \neq 0$.

Several earlier studies have been conducted on the subject of correlation between bond and stock prices and rates of return. For example, Barsky (1989) found that real interest rate changes are important in moving the bond and the stock fund prices, and Campbell et al. (1997) found evidence to back this up. They found a significant positive correlation between news about real interest rates and news about equity premiums. Fleming et al. (1998) found that the same information process does not drive the bond and the stock market. On the subject of correlation between the rates of return, Campbell and Ammer (1993) found very low positive correlation in post-war U.S. data, as did Shiller and Beltratti (1992) in U.S. and U.K. data. We on the other hand found a very high correlation between the rates of return but negative instead of positive. However, Stivers and Sun (2002) found that higher stock market uncertainty is associated with little co-movement or even a negative co-movement between stock and bond returns. Our historical data concerns the period December 2000-December 2004, which is a period of high stock market volatility. The negative correlation coefficients we found between the rates of return in the Dutch market are consistent with previous investigations, but they are rather high. This highly negative correlation emphasizes the need to investigate how to price derivative contracts with a mix fund as the underlying asset.

\section{Modeling assumptions and valuation formula}

In this section we will discuss our main modeling assumptions for the stochastic dynamics of the mix fund price process. 
(A1) The mix fund is continuously rebalanced in such a way that a fixed percentage $w_{s}$ of capital is invested in the stock fund, and the rest in the bond fund.

(A2) The stock fund price process follows the Black-Scholes model, with a volatility function $\sigma_{s}(t)$ which may be time-varying.

(A3) The bond market can be modeled by the Hull-White extension of the Vasiček short rate model, and the short rate volatility function $\sigma_{r}(t)$ may be time-varying.

(A4) The bond fund portfolio is continuously rebalanced in a self-financing way to match certain desired values of its interest rate sensitivity, or 'Duration' $D(t)$. These desired values may be time-varying but should be known on beforehand.

We now formulate the model more explicitly. On a filtered probability space $\left(\Omega, \mathcal{F},\left(\mathcal{F}_{t}\right)_{t \geq 0}, P\right)$ which supports two standard $\mathcal{F}_{t}$-adapted Brownian Motion processes $\left\{W_{t}, t \geq 0\right\}$ and $\left\{V_{t}, t \geq 0\right\}$ with correlation coefficient $\rho$ we define zero coupon bond price processes $p(t, T)$ for all times $t \geq 0$ and maturities $T \geq t$ in a Hull-White term structure. We thus have

$$
\mathrm{d} r_{t}=\left(\Theta(t)-a r_{t}\right) \mathrm{d} t+\sigma_{r}(t) \mathrm{d} V_{t}
$$

where the mean-reversion parameter $a>0$, the time-varying short rate volatility function $\sigma_{r}: \mathbb{R}^{+} \rightarrow \mathbb{R}^{+}$and the initial term structure parameter $\Theta: \mathbb{R}^{+} \rightarrow \mathbb{R}$ are all assumed to be known on beforehand. The bond price processes then satisfy (see standard references such as Duffie, 1996)

$$
p(t, T)=A(t, T) \mathrm{e}^{-\beta(t, T) r_{t}}
$$

where

$$
\begin{aligned}
& \beta(t, T)=\frac{1}{a}\left(1-\mathrm{e}^{-a(T-t)}\right), \\
& A(t, T)=\exp \int_{t}^{T}\left(\frac{1}{2} \sigma_{r}^{2}(s) \beta^{2}(s, T)-\Theta(s) \beta(s, T)\right) \mathrm{d} s
\end{aligned}
$$

We assume that our bond portfolio $B$ invests in a self-financing portfolio of $N$ different bonds ${ }^{1}$ with maturities $\left\{T_{1}, T_{2}, \ldots, T_{N}\right\}$ and we denote the amount invested in the bond with maturity $T_{i}$ by $c_{i}(t)$. We now assume that the fund will be managed in such a way that the sensitivity with respect to the short rate is kept at a prescribed (but possibly time-varying) level:

$$
\frac{\sum_{i=1}^{N} c_{i}(t) p\left(t, T_{i}\right) \beta\left(t, T_{i}\right)}{\sum_{j=1}^{N} c_{j}(t) p\left(t, T_{j}\right)}=D(t)
$$

Note that in practice, duration is usually specified as a sensitivity with respect to a certain fixed yield to maturity, but since we have many different maturities, we need to define the sensitivity here with respect to one fixed chosen rate and in a Hull-White model the short rate seems to be the most natural. We then find

$$
\frac{\mathrm{d} B_{t}}{B_{t}}=\mu_{b}\left(t, r_{t}\right) \mathrm{d} t-D(t) \sigma_{r}(t) \mathrm{d} V_{t}
$$

for a function $\mu_{b}: \mathbb{R}^{+} \times \mathbb{R}^{+} \rightarrow \mathbb{R}$ which can be calculated explicitly but which will turn out to be of no importance in the further analysis.

Combining this with a Black-Scholes model for the stock price process

$$
\frac{\mathrm{d} S_{t}}{S_{t}}=\mu_{s}(t) \mathrm{d} t+\sigma_{s}(t) \mathrm{d} W_{t}
$$

\footnotetext{
${ }^{1}$ This means we specify all bonds in which we may invest during the lifetime of the guarantee, so investment in a particular bond may well be zero for certain times.
} 
we can then find the stochastic dynamics of the mix fund, since we assumed that a fixed percentage $w_{s}$ of the capital would be invested in stock and the rest in bonds

$$
\frac{\mathrm{d} M_{t}}{M_{t}}=w_{s} \frac{\mathrm{d} S_{t}}{S_{t}}+\left(1-w_{s}\right) \frac{\mathrm{d} B_{t}}{B_{t}}=\left(\left(1-w_{s}\right) \mu_{b}\left(t, r_{t}\right)+w_{s} \mu_{s}(t)\right) \mathrm{d} t-\left(1-w_{s}\right) D(t) \sigma_{r}(t) \mathrm{d} V_{t}+w_{s} \sigma_{s}(t) \mathrm{d} W_{t}
$$

It will be convenient to work with independent Brownian Motions so we take ${ }^{2}$

$$
\begin{aligned}
& W_{t}=\rho W_{t}^{1}+\sqrt{1-\rho^{2}} W_{t}^{2} \\
& V_{t}=W_{t}^{1}
\end{aligned}
$$

for a two-dimensional Brownian Motion process $\mathbf{W}_{t}=\left(W_{t}^{1}, W_{t}^{2}\right)$, and our market model for the mixed fund and the $T$-bond $P_{t}=p(t, T)$ is thus

$$
\begin{aligned}
\frac{\mathrm{d} M_{t}}{M_{t}} & =\mu_{M}\left(t, r_{t}\right) \mathrm{d} t+\left(\rho w_{s} \sigma_{s}(t)-\left(1-w_{s}\right) D(t) \sigma_{r}(t)\right) \mathrm{d} W_{t}^{1}+\sqrt{1-\rho^{2}} w_{s} \sigma_{s}(t) \mathrm{d} W_{t}^{2} \\
\frac{\mathrm{d} P_{t}}{P_{t}} & =\mu_{P}\left(t, r_{t}\right) \mathrm{d} t-\beta(t, T) \sigma_{r}(t) \mathrm{d} W_{t}^{1}
\end{aligned}
$$

Our goal will now be to price today (time $t=0$ ) a guaranteed rate of return $R_{\min }$ on a nominal value of $M_{0}$ euro over the next $T$ years, i.e. a contingent claim which pays at a certain time of maturity $T>0$ the amount

$$
C_{T}=\max \left(K-M_{T}, 0\right), \quad K=M_{0} \mathrm{e}^{R_{\min } T}
$$

To find the price for such a claim and a corresponding hedging strategy, we would like to find three processes $\left(h_{t}^{S}, h_{t}^{B}, h_{t}^{P}\right)$ which are adapted with respect to the filtration generated by the $\left(S_{t}, r_{t}\right)$ process (i.e. at all times they only make use of information available at that time) such that the price process of the contingent claim $C_{t}$ satisfies $C_{T}=\max \left(K-M_{T}, 0\right)$, while at all times $t \in[0, T]$,

$$
\begin{aligned}
& C_{t}=h_{t}^{S} S_{t}+h_{t}^{B} B_{t}+h_{t}^{P} p(t, T) \\
& \mathrm{d} C_{t}=h_{t}^{S} \mathrm{~d} S_{t}+h_{t}^{B} \mathrm{~d} B_{t}+h_{t}^{P} \mathrm{~d} p(t, T)
\end{aligned}
$$

The first equation means that at all times (and therefore, more particularly, at the final payoff time) the value of our hedging portfolio is worth the same as the guarantee that we have given, while the second equation makes sure that the portfolio is self-financing, i.e. this portfolio can be created by changing our position in $S, B$ and $P$ without injecting extra money.

The correct price and hedging strategy are given by the following theorem, which is proved in Appendix A.

Theorem 3.1. Under the assumptions stated, the price of the mix fund guarantee started at time zero equals, for any time $t \in[0, T[$

$$
\begin{gathered}
M_{0} p(t, T) \mathrm{e}^{R_{\min } T} N\left(\frac{\ln \left(p(t, T) M_{0} / M_{t}\right)+R_{\min } T+\frac{1}{2} \int_{t}^{T} \sigma_{M / P}^{2}(u) \mathrm{d} u}{\sqrt{\int_{t}^{T} \sigma_{M / P}^{2}(u) \mathrm{d} u}}\right) \\
-M_{t} N\left(\frac{\ln \left(p(t, T) M_{0} / M_{t}\right)+R_{\min } T-\frac{1}{2} \int_{t}^{T} \sigma_{M / P}^{2}(u) \mathrm{d} u}{\sqrt{\int_{t}^{T} \sigma_{M / P}^{2}(u) \mathrm{d} u}}\right)
\end{gathered}
$$

where

$$
\sigma_{M / P}^{2}(t)=w_{s}^{2} \sigma_{s}^{2}(t)+2 \rho w_{s}\left[\beta(t, T)-\left(1-w_{s}\right) D(t)\right] \sigma_{r}(t) \sigma_{s}(t)+\left[\beta(t, T)-\left(1-w_{s}\right) D(t)\right]^{2} \sigma_{r}^{2}(t)
$$

\footnotetext{
${ }^{2}$ Note that if $\rho$ is positive, this means positive correlation between the Brownian Motions, hence negative correlation between the stock and bond returns.
} 
The portfolio consisting of $h^{S}$ stocks, $h^{B}$ bonds from the bond fund and $h^{P}$ T-bonds, with

$$
\begin{aligned}
h_{t}^{S} & =w_{s} \frac{M}{S} \frac{\partial C}{\partial M}\left(t, M_{t}, p(t, T)\right) \\
h_{t}^{B} & =\left(1-w_{s}\right) \frac{M}{B} \frac{\partial C}{\partial M}\left(t, M_{t}, p(t, T)\right) \\
h_{t}^{P} & =\frac{\partial C}{\partial P}\left(t, M_{t}, p(t, T)\right)
\end{aligned}
$$

constitutes a replicating strategy for the claim (a delta-hedging strategy), where $M$ denotes the current value of the mix fund, $B$ the value of (one unit in) the bond fund, and $C\left(t, M_{t}, p(t, T)\right)$ is given by Eq. (3.1).

\section{Remarks.}

- The essential property that is exploited here, is the lognormality of the mix fund price process under the $T$-bond numeraire, $M_{t} / p(t, T)$. Results such as the one here have been formulated before (Geman et al., 1995) but we give a simplified proof (without the usual technicalities surrounding the change-of-measure approach used in the earlier papers) by the use of homogeneity methods which were formulated earlier (Hoogland and Neumann, 2001; Hoogland et al., 2003). Changes of measures and changes of numeraires can simply be reduced to rewriting the problem in different coordinates.

- Due to this structure, it easily follows that a time-varying (but known!) mixing strategy $w_{s}(t)$ will give exactly the same formula when $w_{s}$ is replaced by $w_{s}(t)$ in the formulas. The same is true for a time-varying correlation structure $\rho(t)$.

- If the mix fund (or the bond fund which is part of the mix fund) is reduced in price by a continuous or discretely paid 'management fee' then this can easily be incorporated in the model as well, by modeling continuous or discrete dividend payments in the assets.

- A positive $\rho$ means negative correlation between stock and bond returns, so one might expect that this would reduce the volatility, and hence the value of the guarantee. But Eq. (3.2) shows that there are two correlation effects involved: (1) correlation of the stocks with the bonds in the mix fund, which is proportional to $\left(1-w_{s}\right) D(t)$ and decreases the volatility, and (2) correlation of stocks with the stochastic discount factors, which is proportional to $\beta(t, T)$ and increases the volatility. The overall effect of a positive $\rho$ may thus be a higher volatility and therefore a higher price for the guarantee, if $\int_{t}^{T}\left[\beta(u, T)-\left(1-w_{s}\right) D(u)\right] \sigma_{r}(u) \sigma_{s}(u) \mathrm{d} u>0$. This also shows that a positive effect of $\rho$ on the option price at the start of a contract (time zero) may change at a later time during the lifetime of the contract. The positive contribution, proportional to $\beta(t, T)$, starts at a high value but decreases over time since the remaining stochasticity in the discount factor becomes smaller. But the negative contribution, proportional to $\left(1-w_{s}\right) D(t)$, will be constant if we have a constant duration. Close to maturity the negative effect may therefore become larger than the positive effect and we thus see that it can happen that the sensitivity of the guarantee's value with respect to the correlation coefficient $\rho$ may actually change sign during the lifetime of the contract.

If we look at the start of the contract $(t=0)$ and define the initial yield of the $T$-bond at that time as

$$
R_{T}=-\frac{\ln p(0, T)}{T}
$$

then the premium for the guarantee per unit invested in the mix fund equals

$$
\mathrm{e}^{\left(R_{\min }-R_{T}\right) T} N\left(\sqrt{T} \frac{\left(R_{\min }-R_{T}\right)+\frac{1}{2} \bar{\sigma}^{2}}{\bar{\sigma}}\right)-N\left(\sqrt{T} \frac{\left(R_{\min }-R_{T}\right)-\frac{1}{2} \bar{\sigma}^{2}}{\bar{\sigma}}\right)
$$

where $\bar{\sigma}^{2}=\frac{1}{T} \int_{0}^{T} \sigma_{M / P}(u)^{2} \mathrm{~d} u$ (with $\sigma_{M / P}$ as defined in Theorem 3.1), and this shows that the only other relevant parameter is the spread between the currently available yield $R_{T}$ and the guaranteed yield $R_{\min }$. 


\section{Sensitivity analysis}

We perform an experiment in which we use the standard parameter values

$$
a=0.05, \quad \sigma_{r}(t)=0.010, \quad \sigma_{s}(t)=0.25, \quad D=5.0,
$$

and

$$
T=10, \quad R_{T}=4 \%, \quad R_{\min }=2 \%, \quad w_{s}=\frac{1}{2}, \quad \rho=0
$$

The value for these parameters of the guarantee equals $C=0.0726 \%$ at time zero. To investigate the influence of the different parameters on this value, we will keep all parameters fixed apart from two in a number of different combinations.

In the first plot, we vary the yield $R_{T}$ on the $T$-bond (i.e. the bond with the same maturity as the guarantee) and the minimal rate of return guaranteed $R_{\min }$. The value is obviously decreasing in the former and increasing in the latter variable, and in this figure we see the strong curvature in both, especially for high values (Fig. 1).

In the second plot we vary the maturity of the guarantee $T$ (while keeping the yield for that maturity fixed at $4 \%$ ) and the duration of the bonds in the mix fund. For small maturities $T$, the value of the guarantee increases when the duration increases but for larger values of the time to maturity this is no longer the case. The value is then decreasing for low durations and increasing for higher ones, and there is thus a minimum value of the guarantee for a specific duration value (Fig. 2).

This minimum can be calculated exactly: tedious but straightforward calculations establish that if all the parameters are taken to be constant in time, a minimum occurs for $D=D^{*}$ where

$$
D^{*}=\frac{\rho w_{s}}{1-w_{s}} \frac{\sigma_{s}}{\sigma_{r}}+\frac{1}{1-w_{s}} \frac{\mathrm{e}^{-a(T-t)}-1+a(T-t)}{a^{2}(T-t)}
$$

and this is the duration which corresponds to the minimal variance portfolio in a Markowitz sense. The second term in this expression (the only one when bonds and stocks move independently) is close to $\frac{1}{2}(T-t) /\left(1-w_{s}\right)$ for small values of $a(T-t)$ and represents the interest rate sensitivity of the discounting factor used in our guarantee. The first term is due to correlation effects between stocks and bonds. For the parameter values as given above, we have $D^{*}=8.52+25.00 \rho$ so the influence on $D^{*}$ of the correlation coefficient is substantial.

In Fig. 3 we show the direct influence of the correlation coefficient $\rho$ on the value of the guarantee. For different volatilities $\sigma_{s}$ and $\sigma_{r}$ this figure shows those values for $\rho=+\frac{1}{2}, 0$ and $-\frac{1}{2}$. The highest guarantee values represent the case $\rho=+\frac{1}{2}$ in this figure, as explained in the last remark of the previous section. We see that even for moderate

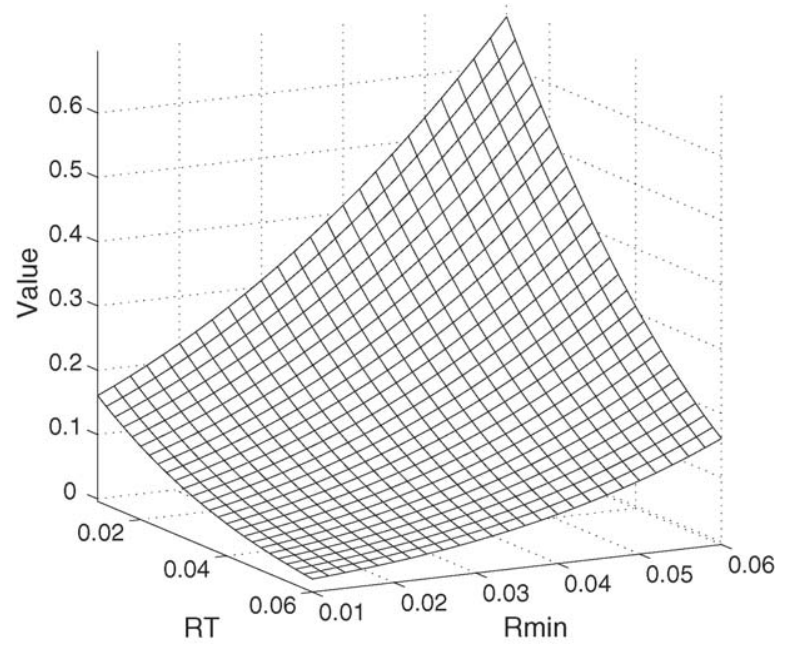

Fig. 1. Value of guarantee as function of $R_{T}$ and $R_{\min }$. 


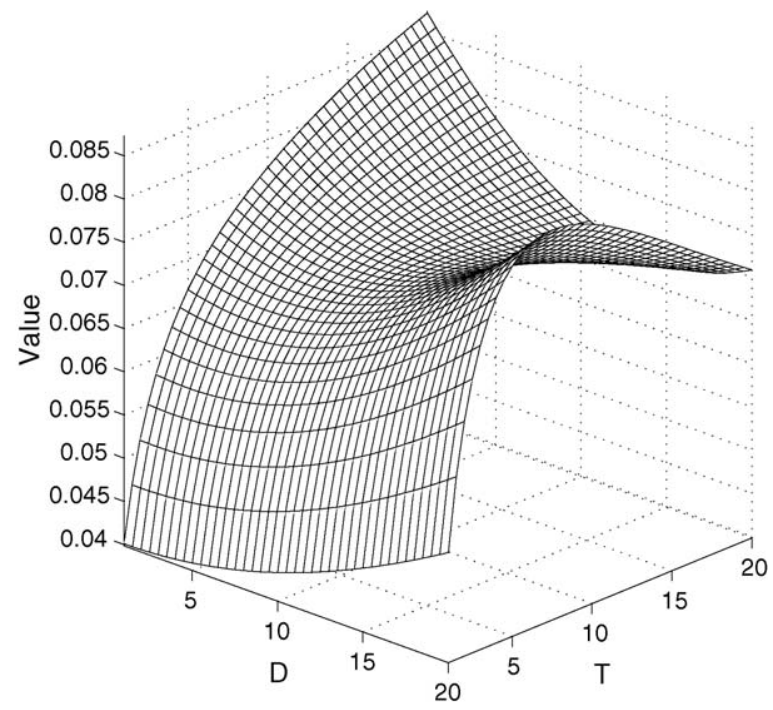

Fig. 2. Value of guarantee as function of $D$ and $T$.

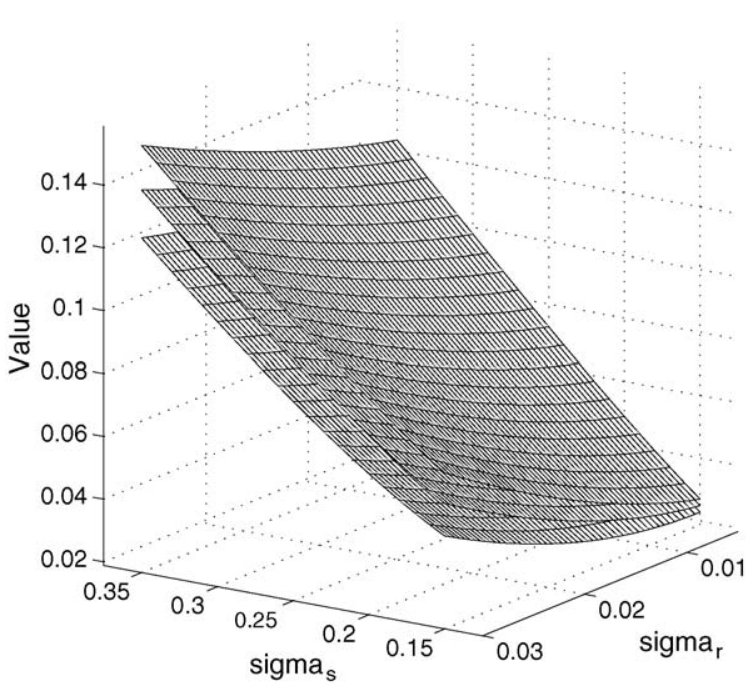

Fig. 3. Value of guarantee as function of $\sigma_{s}$ and $\sigma_{r}$ for different values of $\rho$.

values of $\sigma_{r}$ and $\sigma_{s}$ the differences (as percentage of the guarantee value) become substantial, thus emphasizing the importance of incorporating the correct correlation values in our models.

Fig. 4 shows the influence of the mixing parameter $w_{s}$. We included the duration $D$ in this figure as well, to emphasize the obvious fact that the influence of duration on the guarantee value depends strongly on the relative size of the components in the mix fund: if almost all the assets are invested in stock then the duration of bonds is hardly of any importance.

\section{Premium linked guarantees}

In practice, guarantees are often not given for the rate of return of a single investment over one fixed time interval, but over the rate of return made when a fixed notional amount is invested in the mix fund periodically during a certain time interval. The investment policy thus takes the form of an annuity, and the participation in the mix fund at the end of the investment period thus critically depends on the mix fund prices at the investment dates. If we invest a fixed 


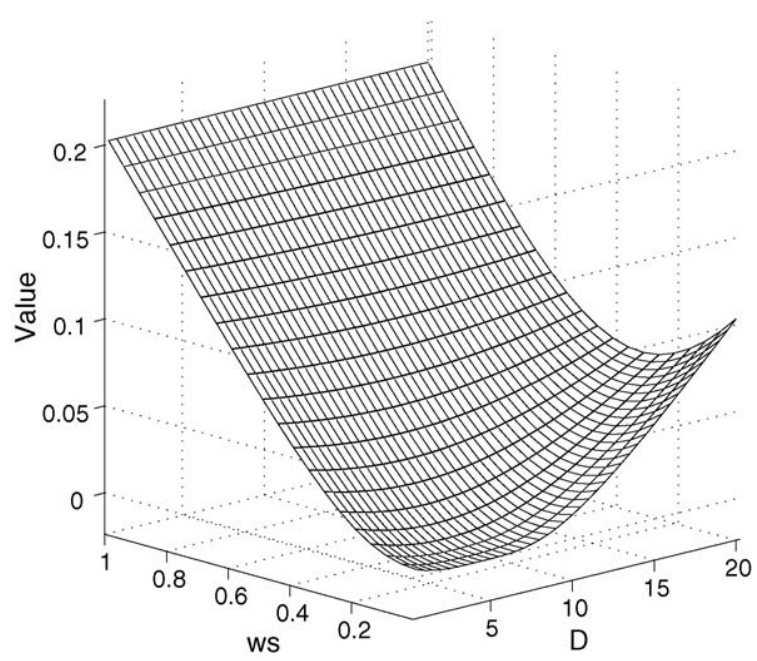

Fig. 4. Value of guarantee as function of $w_{s}$ and $D$.

notional amount $N$ at the times $T_{1}, T_{2}, \ldots, T_{n}$ then the present value of all the money invested equals the annuity value $N \sum_{i=1}^{n} p\left(t, T_{i}\right)$, where $t$ represents the current time. The number of mix fund assets bought at time $T_{i}$ equals $N / M_{T_{i}}$ and the total value of our participation in the mix fund at the guarantee date $T$ thus equals

$$
M_{T} \sum_{i=1}^{n} \frac{N}{M_{T_{i}}}
$$

and since we guarantee that all invested cashflow earns at least the rate of return $R_{\min }$ the payoff of the guarantee at time $T$ equals

$$
N\left(M_{T} \sum_{i=1}^{n} \frac{1}{M_{T_{i}}}-\sum_{i=1}^{n} \mathrm{e}^{R_{\min }\left(T-T_{i}\right)}\right)^{+}
$$

No closed-form formula for the valuation and hedging strategy for such an option exists. One could use similar bounds to the ones derived by Schrager and Pelsser (2004) by extending their results to the mix-fund case, but we can determine the fair value using Monte Carlo simulations as well, since it is known that this fair value equals the expected value under the riskneutral measure. We proceed as follows.

- Take a certain yield curve and some other products (swaptions, caps) which contain information about its volatility, and fit the Hull-White model (i.e. identify estimates for $a, \sigma_{r}$ and use $\theta(t)$ to fit the curve using these parameters).

- Simulate many sample paths for a discretized version of the stochastic differential equations under the riskneutral measure ${ }^{3}$ :

$$
\begin{aligned}
\mathrm{d} r_{t} & =a\left(\Theta(t)-r_{t}\right) \mathrm{d} t+\sigma_{r}(t) \mathrm{d} W_{t}^{1} \\
\frac{\mathrm{d} M_{t}}{M_{t}} & =\mu_{M}\left(t, r_{t}\right) \mathrm{d} t+\left(\rho w_{s} \sigma_{s}(t)-\left(1-w_{s}\right) D(t) \sigma_{r}(t)\right) \mathrm{d} W_{t}^{1}+\sqrt{1-\rho^{2}} w_{s} \sigma_{s}(t) \mathrm{d} W_{t}^{2} \\
\frac{\mathrm{d} P_{t}}{P_{t}} & =\mu_{P}\left(t, r_{t}\right) \mathrm{d} t-\beta(t, T) \sigma_{r}(t) \mathrm{d} W_{t}^{1} \\
\mathrm{~d} H_{t} & =r_{t} H_{t} \mathrm{~d} t
\end{aligned}
$$

\footnotetext{
${ }^{3}$ In fact, for numerical calculations we discretized the dynamics of the logarithm of $P$ and $M$ since we then have enhanced stability and second order convergence.
} 


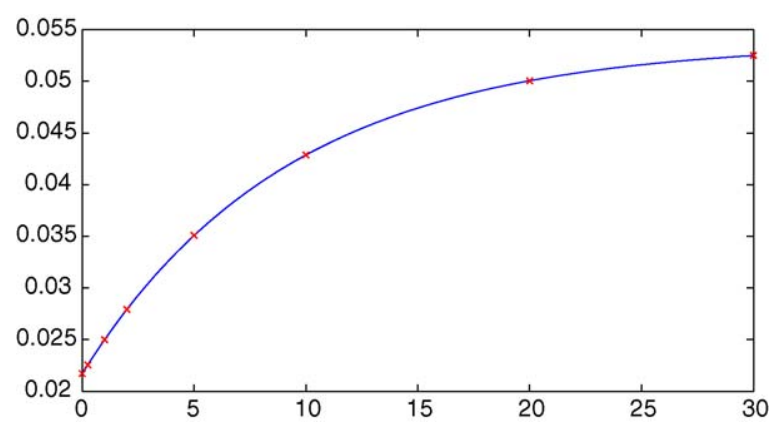

Fig. 5. Yield curve.

The last equation simulates the value of a bank account, which we need for discounting purposes in the next equation.

- On every sample path evaluate the discounted value of the payoff per notional amount $N$ of our guarantee minus the payoff of the guaranteed value for the simpler case we treated in the previous paragraph (the European put option)

$$
\frac{\left(M_{T} \sum_{i=1}^{n}\left(1 / M_{T_{i}}\right)-\sum_{i=1}^{n} \mathrm{e}^{R_{\min }\left(T-T_{i}\right)}\right)^{+}-\left(M_{T}-\mathrm{e}^{R_{\min }(T-t)}\right)^{+}}{H_{T} / H_{t}}
$$

- Take the average value over all sample paths of this value, and then add the theoretical price for the guaranteed value for the simpler case we treated in the previous paragraph.

The reason we generate a Monte Carlo estimate for the price difference between our more complicated case and the easier case treated before (and then compensate for it in the last step) is that the variance of this price difference (and hence the inaccuracy of our estimate) will then be greatly reduced. This also shows the value of finding explicit formulas for the earlier simplified case: it will make our Monte Carlo procedure much more efficient.

As a test case we use an interpolated yield curve shown above (Fig. 5), with parameter values $a=0.050, \sigma_{r}=1.00 \%$ which govern its stochastic movements. We assume we invest a fixed nominal amount equal to 1 at times 0 (today) and years $1,2, \ldots, T-1$ afterwards and we take guaranteed rates of return of $R_{\min }=0 \%, 2 \%$ or $4 \%$ for all the money invested at the final settlement date $T$, which we took 10 or 20 (years).

The mix fund percentages in bonds and stocks vary $\left(w_{s}=0 \%, 25 \%, 50 \%, 75 \%\right.$ or $\left.100 \%\right)$, and the volatility of the stock equals $\sigma_{s}=25 \%$. The bond fund had a fixed duration $D=5$ (years). We simulated 1,500,000 sample paths with 5000 time steps per sample path. The estimated $95 \%$ confidence interval bounds for the Monte Carlo values are less than \pm 0.002 in all cases.

The results can be found in Table 1 (for maturity $T=10$ ) and Table 2 (for maturity $T=20$ ). Note that the values of the guarantee increase with $R_{\min }$ but that for $R_{\min }=0$ the value may sometimes decrease with maturity, since it may be easier to protect against a loss when a longer investment period is considered and the yields for long-term bonds are higher as well.

We also see that if the mix fund consists of bonds alone $\left(w_{s}=0\right)$ then $\rho$ obviously no longer has any influence but it is not true that a mix fund consisting only of stock $\left(w_{s}=1\right)$ becomes independent of $\rho$, since the discount rates will still correlate with the equity price process. In all cases the value of the guarantee grows with the stock participation $w_{s}$ due to the increasing volatility of the mix fund price process.

The prices given here are for the initial time $(t=0)$ value of the contract. As mentioned before in the last remark of Section 3, Eq. (3.2) shows that the term which describes the influence of $\rho$ on the volatility, and hence on the price, is proportional to $w_{s} \int_{t}^{T}\left[\beta(u, T)-\left(1-w_{s}\right) D\right] \mathrm{d} u$. This will usually start with a positive value at $t=0$ but become negative for $t$ close to the maturity date $T$. The only exception here is the case where $T=10$ and $w_{s}=25 \%$, when this sensitivity is already close to zero for $t=0$. But at later times, when we are closer to maturity and the value of $\beta(t, T)$ has decreased, the influence of $\rho$ may change sign, and may now decrease rather than increase the value of the guarantee. 
Table 1

Monte Carlo results for maturity $T=10$

\begin{tabular}{|c|c|c|c|c|c|}
\hline \multirow[t]{2}{*}{$R_{\min }$} & \multicolumn{5}{|l|}{$w_{s}$} \\
\hline & $0 \%$ & $25 \%$ & $50 \%$ & $75 \%$ & $100 \%$ \\
\hline \multicolumn{6}{|c|}{$\rho=0.00$} \\
\hline $0 \%$ & 0.000 & 0.015 & 0.158 & 0.411 & 0.712 \\
\hline $2 \%$ & 0.008 & 0.088 & 0.357 & 0.699 & 1.060 \\
\hline $4 \%$ & 0.139 & 0.343 & 0.725 & 1.132 & 1.540 \\
\hline \multicolumn{6}{|c|}{$\rho=0.15$} \\
\hline $0 \%$ & 0.000 & 0.015 & 0.162 & 0.426 & 0.737 \\
\hline $2 \%$ & 0.008 & 0.087 & 0.364 & 0.717 & 1.089 \\
\hline $4 \%$ & 0.139 & 0.341 & 0.734 & 1.153 & 1.573 \\
\hline \multicolumn{6}{|c|}{$\rho=0.30$} \\
\hline $0 \%$ & 0.000 & 0.014 & 0.167 & 0.440 & 0.761 \\
\hline $2 \%$ & 0.008 & 0.086 & 0.372 & 0.735 & 1.117 \\
\hline $4 \%$ & 0.139 & 0.339 & 0.743 & 1.174 & 1.605 \\
\hline
\end{tabular}

Table 2

Monte Carlo results for maturity $T=20$

\begin{tabular}{|c|c|c|c|c|c|}
\hline \multirow[t]{2}{*}{$R_{\min }$} & \multicolumn{5}{|l|}{$w_{s}$} \\
\hline & $0 \%$ & $25 \%$ & $50 \%$ & $75 \%$ & $100 \%$ \\
\hline \multicolumn{6}{|c|}{$\rho=0.00$} \\
\hline $0 \%$ & 0.000 & 0.008 & 0.118 & 0.410 & 0.825 \\
\hline $2 \%$ & 0.010 & 0.083 & 0.413 & 0.940 & 1.549 \\
\hline $4 \%$ & 0.221 & 0.531 & 1.205 & 1.992 & 2.797 \\
\hline \multicolumn{6}{|c|}{$\rho=0.15$} \\
\hline $0 \%$ & 0.000 & 0.010 & 0.135 & 0.450 & 0.886 \\
\hline $2 \%$ & 0.010 & 0.094 & 0.450 & 1.004 & 1.635 \\
\hline $4 \%$ & 0.221 & 0.561 & 1.268 & 2.081 & 2.907 \\
\hline \multicolumn{6}{|c|}{$\rho=0.30$} \\
\hline $0 \%$ & 0.000 & 0.011 & 0.152 & 0.489 & 0.946 \\
\hline $2 \%$ & 0.010 & 0.104 & 0.487 & 1.066 & 1.717 \\
\hline $4 \%$ & 0.221 & 0.591 & 1.329 & 2.167 & 3.012 \\
\hline
\end{tabular}

\section{Conclusions and suggestions for further research}

In this paper we have provided a framework for the pricing of contracts which guarantee a certain minimal rate of return for participation in a mix fund of stocks and bonds. We have argued that the correlation between stock and bond returns should be taken into account explicitly, and we have also shown the importance of the duration of the bond portfolio in the mix fund for such models. Indeed, the volatility of the mix fund and the price of the guarantee are very dependent on this duration and as such it might even be used by fund managers who write guarantees on their mix funds to control this volatility.

We trust that our framework is simple yet realistic enough to make explicit calculations feasible for practitioners who are confronted with the growing importance of market-based valuation of embedded options in insurance products. Obviously more complicated models, both for the stochastic interest rates and for the stock dynamics, can be built. But we hope to have addressed in this paper the most essential considerations when modeling guarantees on mix funds, and think these should provide a natural starting point for future bigger models as well.

\section{Appendix A}

To prove Theorem 3.1 we will need the following lemma. 
Lemma. In a market with the $N+1$ asset price processes $\mathbf{S}_{t}=\left(S_{t}^{0}, \ldots, S_{t}^{N}\right)$

$$
\mathrm{d} S_{t}^{i}=S_{t}^{i} \mu_{t} \mathrm{~d} t+S_{t}^{i} \vec{\sigma}^{i}(t) \mathrm{d} \vec{W}_{t}
$$

for $i=0, \ldots, N$, with $\vec{W}$ an $N$-dimensional Brownian Motion, ${ }^{4} \mu_{t}$ any adapted stochastic process, and under the assumption that the vectors $\vec{\sigma}^{i}(u)$ are independent at all times $u$, the price at time tof a European option with maturity $T>t$ and payoff function $\Phi\left(\mathbf{S}_{T}\right)$ which is homogeneous of degree one, i.e.

$$
\Phi(\lambda \mathbf{s})=\lambda \Phi(\mathbf{s}) \quad \text { for all } \lambda>0
$$

equals

$$
C(\mathbf{S}, t)=\int_{\mathbb{R}^{N}} \Phi\left(S^{0} \phi\left(\mathbf{z}-\theta^{0}(t, T)\right), \ldots, S^{N} \phi\left(\mathbf{z}-\theta^{N}(t, T)\right)\right) \mathrm{d} \mathbf{z}
$$

where $\phi$ and $\theta$ are defined by

$$
\phi(\mathbf{z})=\frac{\mathrm{e}^{-(1 / 2)\|\mathbf{z}\|^{2}}}{\sqrt{2 \pi}^{N+1}}, \quad \theta^{i}(t, T) \cdot \theta^{j}(t, T)=\int_{t}^{T} \vec{\sigma}^{i}(u) \cdot \vec{\sigma}^{j}(u) \mathrm{d} u
$$

Proof. It is clear that the homogeneity property (A.1) in $\mathbf{S}$ is inherited by the function $C$ defined above, and differentiation of the homogeneity relation with respect to $\lambda$ and substituting $\lambda=1$ gives

$$
C(\mathbf{S}, t)=\sum_{i=0}^{N} \frac{\partial C}{\partial S_{i}}(\mathbf{S}, t) S^{i}
$$

but according to Ito's rule we also have that

$$
\mathrm{d} C(\mathbf{S}, t)=\sum_{i=0}^{N} \frac{\partial C}{\partial S_{i}}\left(\mathbf{S}_{t}, t\right) \mathrm{d} S_{t}^{i}+\frac{\partial C}{\partial t}\left(\mathbf{S}_{t}, t\right) \mathrm{d} t+\frac{1}{2} \sum_{i=0}^{N} \sum_{j=0}^{N}\left(\vec{\sigma}^{i}(t) \cdot \vec{\sigma}_{j}(t)\right) S_{t}^{i} S_{t}^{j} \frac{\partial^{2} C}{\partial S^{i} \partial S^{j}}\left(\mathbf{S}_{t}, t\right) \mathrm{d} t
$$

which means that we have a self-financing portfolio if $C$ satisfies

$$
0=\frac{\partial C}{\partial t}+\frac{1}{2} \sum_{i=0}^{N} \sum_{j=0}^{N}\left(\vec{\sigma}^{i}(t) \cdot \vec{\sigma}_{j}(t)\right) S^{i} S^{j} \frac{\partial^{2} C}{\partial S^{i} \partial S^{j}}
$$

and one can check rather easily (Hoogland et al., 2003) that this is the case for our definition of $C$. Substitution of $t=T$ in the expression for $C$ shows (using homogeneity once more) that $C(\mathbf{S}, T)=\Phi(\mathbf{S})$ for all $\mathbf{S}$ so $C\left(\mathbf{S}_{t}, t\right)$ is a self-financing process which replicates the contingent claim.

Proof of Theorem 3.1. We apply the result of the previous Lemma with $S^{0}=P, S^{1}=M, \Phi(p, m)=\max \{0, K p-m\}$ (since $p=P(t, T)=1$ at $t=T$ so we can indeed write it in homogeneous form) and $\theta_{1}^{P}(t), \theta_{2}^{P}(t), \theta_{1}^{M}(t), \theta_{2}^{M}(t)$ defined by (A.2) with

$$
\begin{array}{ll}
\vec{\sigma}^{P}(t)=\left[-\beta(t, T) \sigma_{r}(t)\right. & 0] \\
\vec{\sigma}^{M}(t)=\left[\rho w_{s} \sigma_{s}(t)-\left(1-w_{s}\right) D(t) \sigma_{r}(t)\right. & \left.\sqrt{1-\rho^{2}} w_{s} \sigma_{s}(t)\right]
\end{array}
$$

The price of the guarantee is then

$$
C(M, P, t)=\int_{\mathbb{R}^{2}} \Phi\left(P \phi\left(z_{1}-\theta_{1}^{P}(t, T)\right) \phi\left(z_{2}-\theta_{2}^{P}(t, T)\right), M \phi\left(z_{1}-\theta_{1}^{M}(t, T)\right) \phi\left(z_{2}-\theta_{2}^{M}(t, T)\right)\right) \mathrm{d} z_{1} \mathrm{~d} z_{2}
$$

and we now use homogeneity to rewrite this as

$$
C(M, P, t)=P \int_{\mathbb{R}^{2}} \phi\left(z_{1}-\theta_{1}^{P}(t, T)\right) \phi\left(z_{2}-\theta_{2}^{P}(t, T)\right) \Phi\left(1, \frac{M \phi\left(z_{1}-\theta_{1}^{M}(t, T)\right) \phi\left(z_{2}-\theta_{2}^{M}(t, T)\right)}{P \phi\left(z_{1}-\theta_{1}^{P}(t, T)\right) \phi\left(z_{2}-\theta_{2}^{P}(t, T)\right)}\right) \mathrm{d} z_{1} \mathrm{~d} z_{2}
$$

\footnotetext{
${ }^{4}$ Note: This means that the components of the Brownian Motion are independent.
} 
and taking new coordinates $y_{i}=z_{i}-\theta_{i}^{P}(t, T)$ for $i=1,2$ we find

$$
\begin{aligned}
C(M, P, t) & =P \int_{\mathbb{R}^{2}} \phi\left(y_{1}\right) \phi\left(y_{2}\right) \Phi\left(1, \frac{M \phi\left(y_{1}-\theta_{1}^{M}(t, T)+\theta_{1}^{P}(t, T)\right) \phi\left(y_{2}-\theta_{2}^{M}(t, T)+\theta_{2}^{P}(t, T)\right)}{P \phi\left(y_{1}\right) \phi\left(y_{2}\right)}\right) \mathrm{d} y_{1} \mathrm{~d} y_{2} \\
& =P \int_{\mathbb{R}^{2}} \phi\left(y_{1}\right) \phi\left(y_{2}\right) \Phi\left(1, \frac{M}{P} \mathrm{e}^{g\left(y_{1}, y_{2}\right)}\right) \mathrm{d} y_{1} \mathrm{~d} y_{2}
\end{aligned}
$$

where

$$
\begin{aligned}
g\left(y_{1}, y_{2}\right)= & y_{1}\left(\theta_{1}^{M}(t, T)-\theta_{1}^{P}(t, T)\right)+y_{2}\left(\theta_{2}^{M}(t, T)-\theta_{2}^{P}(t, T)\right) \\
& -\frac{1}{2}\left(\theta_{1}^{M}(t, T)-\theta_{1}^{P}(t, T)\right)^{2}-\frac{1}{2}\left(\theta_{2}^{M}(t, T)-\theta_{2}^{P}(t, T)\right)^{2}
\end{aligned}
$$

but when $y_{1}$ and $y_{2}$ have independent standard Gaussian distributions (i.e. $N(0,1)$ distributions) then $g\left(y_{1}, y_{2}\right)$ has a standard Gaussian distribution with mean

$$
\begin{aligned}
- & \frac{1}{2}\left(\theta_{1}^{M}(t, T)-\theta_{1}^{P}(t, T)\right)^{2}-\frac{1}{2}\left(\theta_{2}^{M}(t, T)-\theta_{2}^{P}(t, T)\right)^{2} \\
& =-\frac{1}{2}\left(\theta^{M} \cdot \theta^{M}\right)+\left(\theta^{M} \cdot \theta^{P}\right)-\frac{1}{2}\left(\theta^{P} \cdot \theta^{P}\right) \\
& =-\frac{1}{2} \int_{t}^{T}\left(\vec{\sigma}^{M}(u) \cdot \vec{\sigma}^{M}(u)-2 \vec{\sigma}^{M}(u) \cdot \vec{\sigma}^{P}(u)+\vec{\sigma}^{P}(u) \cdot \vec{\sigma}^{P}(u)\right) \mathrm{d} u=-\frac{1}{2} \int_{t}^{T} \sigma_{M / P}(u)^{2} \mathrm{~d} u
\end{aligned}
$$

where we have used (A.2), and with variance

$$
\left(\theta_{1}^{M}(t, T)-\theta_{1}^{P}(t, T)\right)^{2}+\left(\theta_{2}^{M}(t, T)-\theta_{2}^{P}(t, T)\right)^{2}=\int_{t}^{T} \sigma_{M / P}(u)^{2} \mathrm{~d} u
$$

where we have used (A.2) again. We write

$$
\tilde{\sigma}^{2}=\frac{1}{T-t} \int_{t}^{T} \sigma_{M / P}(u)^{2} \mathrm{~d} u
$$

and we then have

$$
\begin{aligned}
C(M, P, t) & =P \int_{\mathbb{R}} \phi(y) \Phi\left(1, \frac{M}{P} \mathrm{e}^{y \tilde{\sigma} \sqrt{T-t}-(1 / 2) \tilde{\sigma}^{2}(T-t)^{2}}\right) \mathrm{d} y \\
& =P \int_{\mathbb{R}} \frac{\mathrm{e}^{-(1 / 2) y^{2}}}{\sqrt{2 \pi}}\left(\frac{M}{P} \mathrm{e}^{y \tilde{\sigma} \sqrt{T-t}-(1 / 2) \tilde{\sigma}^{2}(T-t)^{2}}-K\right)^{+} \mathrm{d} y=P\left(K N\left(-d_{2}\right)-\frac{M}{P} N\left(-d_{1}\right)\right)
\end{aligned}
$$

with

$$
d_{1}=\frac{\ln \left(M_{t} / K p(t, T)\right)+(1 / 2) \tilde{\sigma}^{2}(T-t)}{\tilde{\sigma} \sqrt{T-t}}, \quad d_{2}=d_{1}-\tilde{\sigma} \sqrt{T-t}
$$

and substitution of $K=M_{0} \mathrm{e}^{R_{\min } T}$ now gives the price result as stated. One can then easily check by some algebra (and using homogeneity) that

$$
\begin{aligned}
& C=h_{S} S+h_{B} B+h_{P} P \\
& \mathrm{~d} C=h_{S} \mathrm{~d} S+h_{B} \mathrm{~d} B+h_{P} \mathrm{~d} P
\end{aligned}
$$

which proves the correctness of the hedging strategy. 


\section{References}

Ernst \& Young, 2002. Embedded Options and Guarantees—Leading-Edge Approaches. Ernst \& Young, Global Insurance Services.

Barsky, R.B., 1989. Why don't prices of stocks and bonds move together? The American Economic Review 79 (5), 1132-1145.

Campbell, J.Y., Ammer, J., 1993. What moves the stock and bond markets? A variance decomposition for long-term asset returns. The Journal of Finance 48 (1), 3-38.

Campbell, J.Y., Lo, A.W., MacKinley, A.C., 1997. The Econometrics of Financial Markets. Princeton University Press, NJ, USA.

Duffie, D., 1996. Dynamic Asset Pricing Theory. Princeton University Press, NJ, USA.

Fabozzi, F.J., 1996. Bond Markets, Analysis and Strategies, third ed. Prentice-Hall, NJ, USA.

Fleming, J., Kirby, C., Ostdiek, B., 1998. Information and volatility linkages in the stock, bond, and money markets. Journal of Financial Economics 49 (1), 111-137.

Geman, H., El Karoui, N., Rochet, J.C., 1995. Changes of numéraire, changes of probability measure and option pricing. Journal of Applied Probability 443-458.

Hoogland, J.K., Neumann, C.D.D., 2001. Local scale invariance and contingent claim pricing I. International Journal of Theoretical and Applied Finance 4 (1), 1-21.

Hoogland, J.K., Neumann, C.D., Vellekoop, M.H., 2003. Symmetries in jump-diffusion models with applications in option pricing and credit risk. International Journal of Theoretical and Applied Finance 6 (2), 135-172.

Nielsen, J.A., Sandmann, K., 2002. Pricing of Asian exchange options under stochastic interest rates as a sum of options. Finance and Stochastics 6, 355-370.

Schrager, D.F., Pelsser, A.A.J., 2004. Pricing rate of return guarantees in regular premium unit linked insurance. Insurance: Mathematics and Economics 35, 369-398.

Shiller, R.J., Beltratti, A.E., 1992. Stock prices and bond yields: can their co-movements be explained in terms of present value models? Journal of Monetary Economics 30 (1), 25-46.

Stivers C., Sun L., 2002. Stock Market Uncertainty and the Relation between Stock and Bond Returns, FRB working paper 2002-3, Atlanta.

Thompson, G.W.P., 1999. Fast Narrow Bounds on the Value of Asian Options. Center for Financial Research, University of Cambridge, preprint.

Vasiček, O., 1977. An equilibrium characterization of the term structure. Journal of Financial Economics 5, 177-188. 